\title{
Central Sensitization in Neurological, Psychiatric, and Pain Disorders: A Multicenter Case-Controlled Study
}

\author{
Keisuke Suzuki (D), ${ }^{1}$ Yasuo Haruyama, ${ }^{2}$ Gen Kobashi, ${ }^{2}$ Toshimi Sairenchi, ${ }^{2}$ \\ Koji Uchiyama $\mathbb{D}^{\mathbb{D}},{ }^{3}$ Shigeki Yamaguchi, ${ }^{4}$ and Koichi Hirata ${ }^{1}$ \\ ${ }^{1}$ Department of Neurology, Dokkyo Medical University, Mibu, Japan \\ ${ }^{2}$ Department of Public Health, Dokkyo Medical University School of Medicine, Mibu, Japan \\ ${ }^{3}$ Laboratory of International Environmental Health, Center for International Cooperation, \\ Dokkyo Medical University, Mibu, Japan \\ ${ }^{4}$ Department of Anesthesiology, Dokkyo Medical University School of Medicine, Mibu, Japan \\ Correspondence should be addressed to Keisuke Suzuki; keisuke@dokkyomed.ac.jp
}

Received 26 November 2020; Revised 29 January 2021; Accepted 1 February 2021; Published 16 February 2021

Academic Editor: Parisa Gazerani

Copyright ( $\odot 2021$ Keisuke Suzuki et al. This is an open access article distributed under the Creative Commons Attribution License, which permits unrestricted use, distribution, and reproduction in any medium, provided the original work is properly cited.

\begin{abstract}
Background. The role of central sensitization in refractory pain-related diseases has not yet been clarified. Methods. We performed a multicenter case-controlled study including 551 patients with various neurological, psychological, and pain disorders and 5,188 healthy controls to investigate the impact of central sensitization in these patients. Symptoms related to central sensitization syndrome (CSS) were assessed by the Central Sensitization Inventory (CSI) parts A and B. Patients were categorized into 5 groups based on CSI-A scores from subclinical to extreme. The Brief Pain Inventory (BPI), addressing pain severity and pain interference with daily activities, and the Patient Health Questionnaire (PHQ)-9, assessing depressive symptoms, were also administered. Results. CSI-A scores and CSI-B disease numbers were significantly greater in patients than in controls $(p<0.001)$. Medium effect sizes $(r=0.37)$ for CSI-A scores and large effect sizes $(r=0.64)$ for CSI-B disease numbers were found between patients and control groups. Compared with the CSI-A subclinical group, the CSI-A mild, moderate, severe, and extreme groups had significantly higher BPI pain interference and severity scores, PHQ-9 scores, and CSS-related disease numbers based on ANCOVA. Greater CSI-B numbers resulted in higher CSI-A scores $(p<0.001)$ and a higher odds ratio ( $p$ for trend $<0.001)$. CSS-related symptoms were associated with pain severity, pain interference with daily activities, and depressive symptoms in various pain-related diseases. Conclusions. Our findings suggest that CSS may participate in these conditions as common pathophysiology.
\end{abstract}

\section{Introduction}

Central sensitization represents enhanced functions of circuits in nociceptive pathways and an abnormal state of the nociceptive systems, resulting from the remarkable plasticity of the somatosensory nervous system [1]. Due to alterations in the somatosensory system from high- to low-threshold pain hypersensitivity in this condition, central sensitization pain occurs in the absence of noxious stimuli. Central sensitization is characterized by allodynia in which pain is induced by nonnoxious stimuli [2], hyperalgesia [3, 4], and widespread pain [5], which was described initially only in animal models $[6,7]$. Central sensitization is defined as increased responsiveness of nociceptive neurons in the central nervous system to their normal or subthreshold afferent input according to the International Association for the Study of Pain [8]. It should be noted that because direct electrophysiological recordings from central nervous systems are not performed in humans, human sensory profiling relies on proxies, which are believed to reflect central sensitization.

Central sensitization plays a role in fibromyalgia, a refractory pain disease in which alteration of central nociceptive processing occurs and pain can be worsened by psychological factors [9]. In patients with migraine, impaired descending pain inhibitory control assessed by 
conditioned pain modulation has been reported in an experimental pain setting compared with healthy controls [10], and central sensitization may contribute to acute allodynia and headache chronification [11]. In patients with restless legs syndrome, which is characterized by an abnormal, often painful sensation in the legs, levodopa-responsive hyperalgesia is thought to be mediated by central sensitization to $\mathrm{A}$ beta-fibers [12]. Patients with irritable bowel syndrome, a common gastrointestinal disorder, show extraintestinal symptoms suggestive of a central hyperalgesic state and have cutaneous hyperalgesia observed in other chronic pain diseases, which is abnormal processing of central nociception [13]. Chang and Lu suggested that irritable bowel syndrome and migraine share many similarities in associated comorbidities and possible pathogenesis regarding pain characteristics and treatment, indicating that they could be the same spectrum disorder involving central sensitization [14]. Central sensitization may be related to a relationship among migraine, inflammatory diseases, and psychiatric disorders [15]; additionally, a relationship among epilepsy, migraine, and psychiatric disorders [16] and a possible role of central sensitization on their pathophysiology have been discussed. A systematic review on central sensitization in chronic whiplash showed that hypersensitivity of the central nervous system plays an important role in persisting pain complaints in patients with chronic whiplash, but the underlying mechanisms are unclear [17].

Based on these findings, central sensitization plays a key role in sustained and amplified pain observed in certain proportions of refractory pain-related diseases, and it can participate in the pathophysiology of pain chronification, contributing to pain-related disability and inducing comorbid psychiatric symptoms and nonspecific symptoms such as fatigue and dizziness. These consequences not only negatively impact daily activity and the quality of life of affected individuals but also reduce social productivity. However, the role of central sensitization in refractory painrelated diseases is not yet fully understood. Investigating the effect of central sensitization in various diseases requires a large sample of pain-related diseases with a multicenter collaboration.

The Central Sensitization Inventory (CSI) is a self-administered questionnaire addressing symptoms associated with central sensitization and screening for several diseases related to central sensitization, including chronic headaches, restless legs syndrome, fibromyalgia, chronic fatigue syndrome, and multiple chemical sensitivity. CSI has been widely used and validated for the assessment of symptoms related to central sensitization syndrome (CSS) [18]. In this study, we performed a multicenter study using the Japanese version of the CSI [19] to assess symptoms related to central sensitization in patients with neurological, psychological, and pain disorders.

\section{Methods}

2.1. Participants. A total of 567 outpatients (199 M/368 F; age, $57.6 \pm 18.1$ years) were recruited from neurological psychiatric pain clinics in a multicenter setting between
March 2018 and April 2019. Patient diagnoses in this multicenter study included migraine, tension-type headache, medication overuse headache, Parkinson's disease, stroke, restless legs syndrome, neuropathy, fibromyalgia, complex regional pain syndrome, cervical/lumbar spine disease, chronic fatigue syndrome, and depression. After excluding missing data on CSI, 551 outpatients $(193 \mathrm{M}$ / $358 \mathrm{~F}$; age $57.1 \pm 18.0$ years) were finally included. Additionally, 6,135 residents as general healthy controls were recruited from Utsunomiya city located adjacent to Dokkyo Medical University Hospital. When they had undergone an annual health check-up, they answered the questionnaire. Excluding missing values for sex, age, and CSI, 5,188 $(2,036 \mathrm{M} / 3,152 \mathrm{~F}$; age $64.7 \pm 12.1$ years $)$ were included in this study.

The present study was carried out in accordance with the Declaration of Helsinki and was approved by the institutional review boards of Dokkyo Medical University Hospital and other participating facilities. All participants provided written informed consent to participate in the study.

2.2. Clinical Assessments. Participants completed questionnaires including smoking, caffeine intake, alcohol consumption, and body mass index. CSS-related symptoms were assessed by the Japanese version of the CSI, comprising parts A and B [19]. CSI-A scores 25 items for health-related and CSS-related somatic symptoms (score, 0-100), and CSIB screens whether subjects have previously been diagnosed with 10 specific CSS diagnoses: restless legs syndrome, chronic fatigue syndrome, fibromyalgia, temporomandibular joint disorder, migraine or tension headaches, irritable bowel syndrome, and multiple chemical sensitivity. Cronbach's $\alpha$ coefficients of CSI-A scores were 0.902 in the patient group and 0.894 in the control group in the present study. Patients were categorized into 5 groups based on CSI-A scores: subclinical, 0-29; mild, 30-39; moderate, 40-49; severe, 50-59; and extreme, 60-100 [19, 20]. A CSI-A score of $\geq 40$ is reportedly the optimal cutoff for distinguishing subjects with CSS and those without [19]. Patients completed the Brief Pain Inventory (BPI) Japanese version, consisting of a pain severity score (means of items 3-6), assessing pain severity, and a pain interference score (mean of items 9A-9G), addressing pain interference with daily activities [21]. The Japanese version of the Patient Health Questionnaire (PHQ)-9, assessing depressive symptoms (scores, 0-27), was also completed [22].

2.3. Statistical Analysis. Patient and control groups were compared using the chi-square test or Fisher's exact test for categorical variables and Student's $t$-test or Mann-Whitney $U$ test for continuous variables where appropriate. To compare the CSI-A score and CSI prevalence (CSI-A $\geq 40$ points) between patients and control groups, a matching dataset was obtained using caliper matching with a standard deviation of 0.25 times the propensity score calculated by age, gender, smoking, alcohol, and caffeine intake. The effect sizes between the patient and control groups were calculated by formula (1) ( $r \geq 0.1$ : small, $\geq 0.3$ : medium, $\geq 0.5$ : large $)$ for 
TABLE 1: Characteristics of the patient and healthy control group.

\begin{tabular}{|c|c|c|c|c|c|c|c|}
\hline & \multicolumn{3}{|c|}{ Patient group } & \multicolumn{3}{|c|}{ Control group } & \multirow{2}{*}{$p$ value $^{a}$} \\
\hline & $N$ & $(n)$ mean & (\%) SD & $N$ & $(n)$ mean & (\%) SD & \\
\hline Sex, female, $n(\%)$ & 551 & 358 & 65.0 & 5188 & 3152 & 60.8 & 0.053 \\
\hline Age, mean, SD (yr.) & 551 & 57.1 & 18.0 & 5188 & 64.7 & 12.1 & $<0.001$ \\
\hline BMI, mean, SD $\left(\mathrm{kg} / \mathrm{m}^{2}\right)$ & 530 & 23.2 & 4.1 & & - & & \\
\hline Smoking, yes, $n(\%)$ & 542 & 99 & 18.3 & 5035 & 325 & 6.5 & $<0.001$ \\
\hline Alcohol intake, yes, $n(\%)$ & 538 & 225 & 41.8 & 4731 & 1328 & 28.1 & $<0.001$ \\
\hline Caffeine intake, yes, $n(\%)$ & 543 & 501 & 92.3 & 4124 & 2835 & 68.7 & $<0.001$ \\
\hline CSI-A score, mean, SD, points & 551 & 28.1 & 16.0 & 5188 & 15.8 & 11.8 & $<0.001^{\mathrm{b}}$ \\
\hline$\geq 40$ points, $n(\%)$ & & 114 & 20.7 & & 242 & 4.7 & $<0.001$ \\
\hline CSI-B, mean, SD, number of 10 diseases & 551 & 0.99 & 1.14 & 5188 & 0.01 & 0.09 & $<0.001^{\mathrm{b}}$ \\
\hline Migraine or tension headaches, yes, $n(\%)$ & & 234 & 42.5 & & 5 & 0.1 & $<0.001$ \\
\hline Neck injury (including whiplash), yes, $n$ (\%) & & 64 & 11.6 & & 0 & 0.0 & $<0.001^{\mathrm{b}}$ \\
\hline Depression, yes, $n(\%)$ & & 61 & 11.1 & & 35 & 0.7 & $<0.001$ \\
\hline Restless legs syndrome, yes, $n(\%)$ & & 43 & 7.8 & & 0 & 0.0 & $<0.001^{\mathrm{b}}$ \\
\hline Temporomandibular joint disorder, yes, $n(\%)$ & & 40 & 7.3 & & 0 & 0.0 & $<0.001^{\mathrm{b}}$ \\
\hline Anxiety or panic attacks, yes, $n(\%)$ & & 39 & 7.1 & & 0 & 0.0 & $<0.001^{\mathrm{b}}$ \\
\hline Irritable bowel syndrome, yes, $n(\%)$ & & 30 & 5.4 & & 2 & 0.0004 & $<0.001^{\mathrm{b}}$ \\
\hline Fibromyalgia, yes, $n(\%)$ & & 15 & 2.7 & & 0 & 0.0 & $<0.001^{\mathrm{b}}$ \\
\hline Chronic fatigue syndrome, yes, $n(\%)$ & & 14 & 2.5 & & 0 & 0.0 & $<0.001^{\mathrm{b}}$ \\
\hline Multiple chemical sensitivities, yes, $n$ (\%) & & 5 & 0.9 & & 0 & 0.0 & $0.031^{\mathrm{b}}$ \\
\hline \multicolumn{8}{|l|}{ BPI score, mean, SD, points } \\
\hline Pain interference score & 501 & 2.75 & 2.69 & & - & & \\
\hline Pain severity score & 516 & 2.83 & 2.28 & & - & & \\
\hline PHQ score, mean, SD, points & 550 & 13.7 & 6.1 & & - & & \\
\hline
\end{tabular}

BMI: body mass index; CSI: Central Sensitization Inventory; BPI: Brief Pain Inventory; PHQ: Patient Health Questionnaire. ${ }^{a}$ Using a chi-square test or Student's $t$-test; ' ${ }^{\text {b }}$ sing Fisher's exact test or a Mann-Whitney $U$ test.

the Mann-Whitney $U$ test or formula (2) $(\varphi \geq 0.1$ : small, $\geq 0.3$ : medium, $\geq 0.5$ : large) for the chi-square test as follows formulas [23]:

$$
\text { effect } \operatorname{sizes}(\gamma)=\frac{Z}{\sqrt{n}}
$$

where $Z$ is the standard test statics from the Mann-Whitney $U$ test and $n$ is the sample size.

$$
\operatorname{effect} \operatorname{sizes}(\varphi)=\sqrt{\frac{x^{2}}{n(M-1)}},
$$

where $x^{2}$ is chi-square value, $n$ is sample size, $M$ is the value with the fewest rows and columns.

For the patient group, one-way analysis of variance or the Kruskal-Wallis test was used for the analysis of continuous variables among groups where appropriate, and the chi-square test was used for the analysis of categorical variables. Post hoc tests with residual analysis and Benjamini-Hochberg test for a chi-square test and Bonferroni test for ANOVA or Kruskal-Wallis test were performed. The PHQ-9 score, BPI pain interference score, BPI pain severity score, and the number of CSS-related diseases (based on CSI-B) among the 5 groups classified by CSI-A scores were analyzed using analysis of covariance (ANCOVA) after adjustment for potential confounding factors followed by the post hoc Bonferroni test. The relationship between a number of CSS-related diseases (0-6) and CSI-A scores was also analyzed by ANCOVA followed by the Bonferroni test and multivariable logistic regression model after adjustment for potential confounding factors. Each missing value was excluded in every analysis. Two-tailed $p$ values $<0.05$ were considered statistically significant. IBM SPSS Statistics software version 24.0 (IBM SPSS, Inc., Tokyo, Japan) was used for statistical analyses.

\section{Results}

Table 1 shows the characteristics of the patient and control groups in this study. Among the patient groups, the mean CSI-A score was $28.1 \pm 16.0$, and $20.7 \%$ had a CSI-A score $\geq 40$. Among the 10 CSS-related diseases (CSI-B), migraine or tension headache was the most frequent (42.5\%), followed by neck injury (11.6\%), depression (11.1\%), restless legs syndrome (7.8), and so on. Compared with the control group, the patient group had a significantly greater CSI-A score and a number of CSS-related diseases on CSI-B $(p<0.001)$. Medium effect sizes $(r=0.37)$ for CSI-A scores and large effect sizes $(r=0.64)$ for CSI-B disease numbers were found between patients and control groups. Next, we performed a subanalysis comparing 526 patients and 523 propensity score-matched controls. The results replicated significant differences in CSI-A scores and CSSrelated diseases on CSI-B among the groups (Table 2). The effect sizes for the CSI-A score and CSI-B were 0.37 and 0.64 , respectively (Table 2 ).

There was a significant difference in age, sex, number of CSS-related diseases on CSI-B, BPI pain interference score, BPI pain severity score, and PHQ-9 score among the 5 groups classified according to CSI-A scores (Table 3). Post hoc tests showed that the proportion of women was higher in the severe group than in the subclinical score group. The 
TABLE 2: Central Sensitization Inventory of the propensity score-matched patient and control groups.

\begin{tabular}{|c|c|c|c|c|c|c|}
\hline & \multicolumn{2}{|c|}{ Patient group $(n=526)$} & \multicolumn{2}{|c|}{ Control group $(n=523)$} & \multirow{2}{*}{$p$ value $^{\mathrm{a}}$} & \multirow{2}{*}{ Effect size $^{c}$} \\
\hline & $(n)$ mean & $(\%) \mathrm{SD}$ & $(n)$ mean & $(\%) \mathrm{SD}$ & & \\
\hline Sex, female, $n(\%)$ & 338 & 64.3 & 343 & 65.5 & 0.653 & - \\
\hline Age, mean, SD (yr.) & 57.2 & 17.7 & 58.7 & 14.3 & 0.132 & - \\
\hline Smoking, yes, $n(\%)$ & 97 & 18.4 & 108 & 20.7 & 0.367 & - \\
\hline Alcohol intake, yes, $n(\%)$ & 221 & 42.0 & 206 & 39.4 & 0.387 & - \\
\hline Caffeine intake, yes, $n(\%)$ & 488 & 92.3 & 493 & 94.3 & 0.328 & - \\
\hline CSI-A score, mean, SD, points & 28.1 & 15.7 & 17.2 & 12.3 & $<0.001^{\mathrm{b}}$ & 0.37 \\
\hline$\geq 40$ points, $n(\%)$ & 105 & 20.0 & 29.0 & 5.5 & $<0.001$ & 0.22 \\
\hline CSI-B, mean, SD, number of 10 diseases & 1.01 & 1.15 & 0.01 & 0.123 & $<0.001^{\mathrm{b}}$ & 0.64 \\
\hline
\end{tabular}

The propensity score was calculated by age, sex, smoking, alcohol, and caffeine intake. CSI: Central Sensitization Inventory. ${ }^{a}$ Using a chi-square test or

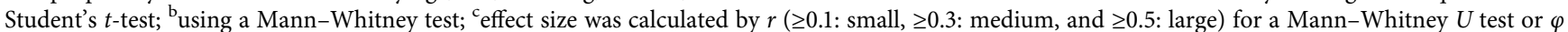
( $\geq 0.1$ : small, $\geq 0.3$ : medium, and $\geq 0.5$ : large) for a chi-square test.

TABLE 3: Differences in each demographic and clinical parameter among the five CSI-A groups.

\begin{tabular}{|c|c|c|c|c|c|c|c|c|c|c|c|c|c|}
\hline \multirow[b]{2}{*}{ Sex, female, $n(\%)$} & \multirow{2}{*}{$\frac{N^{c}}{551}$} & \multicolumn{2}{|c|}{$\begin{array}{l}\text { Subclinical (i) } \\
\quad(0-29)\end{array}$} & \multicolumn{2}{|c|}{$\begin{array}{l}\text { Mild } \\
\text { (ii) }(30-39)\end{array}$} & \multicolumn{2}{|c|}{$\begin{array}{l}\text { Moderate } \\
\text { (iii) }(40-49)\end{array}$} & \multicolumn{2}{|c|}{$\begin{array}{c}\text { Severe } \\
\text { (iv) }(50-59)\end{array}$} & \multicolumn{2}{|c|}{$\begin{array}{l}\text { Extreme } \\
\text { (v) }(60-100)\end{array}$} & \multirow{2}{*}{$\frac{p \text { value }^{\mathrm{a}}}{0.049}$} & \multirow{2}{*}{$\begin{array}{c}\text { Post hoc test } \\
\text { i vs. iv }\end{array}$} \\
\hline & & 192 & 61.0 & 82 & 67.2 & 36 & 69.2 & 33 & 84.6 & 15 & 65.2 & & \\
\hline Age, yr., $n$ mean (SD) & 551 & 315 & $\begin{array}{c}59.8 \\
(17.9)\end{array}$ & 122 & $\begin{array}{c}53.7 \\
(18.6)\end{array}$ & 52 & $\begin{array}{c}53.6 \\
(15.9)\end{array}$ & 39 & $\begin{array}{c}50.8 \\
(16.0)\end{array}$ & 23 & $\begin{array}{c}57.3 \\
(18.9)\end{array}$ & 0.001 & i vs. ii, iv \\
\hline BMI $\left(\mathrm{kg} / \mathrm{m}^{2}\right), n$ mean $(\mathrm{SD})$ & 530 & 303 & $22.9(4.0)$ & 117 & $23.3(3.7)$ & 49 & $23.8(4.7)$ & 38 & $23.9(4.0)$ & 23 & $\begin{array}{l}24.1 \\
(5.6)\end{array}$ & 0.269 & - \\
\hline Smoking, $n(\%)$ & 542 & 49 & 15.7 & 24 & 20.2 & 14 & 27.5 & 8 & 22.2 & 4 & 17.4 & 0.288 & - \\
\hline Alcol & & 131 & & 56 & & 20 & 41 & 12 & & 5 & & & - \\
\hline Caffeine & & 283 & & 114 & & 49 & & 37 & & 18 & & & - \\
\hline $\begin{array}{l}\text { CSS-related diseases on CSI-B, } n \text {, } \\
\text { mean (SD) }\end{array}$ & 551 & 315 & $0.6(0.7)$ & 122 & $1.1(1.0)$ & 52 & $1.4(1.3)$ & 39 & $2.1(1.5)$ & 23 & $2.0(1.4)$ & $<0.001^{\mathrm{b}}$ & $\begin{array}{l}\text { i vs. ii, iii, iv, v } \\
\text { ii vs. iv }\end{array}$ \\
\hline Restless legs syndrome & & 15 & 4.8 & 12 & 9.8 & 5 & 9.6 & 8 & 20.5 & 3 & 13.0 & 0.006 & i vs. iv \\
\hline Chro & & 1 & & 2 & 1 & 2 & & 8 & & 1 & & $<0$ & i vs. iv \\
\hline Fibro & & 0 & 0.0 & 3 & 2.5 & 3 & 5.8 & 8 & 20.5 & 1 & 4.3 & $<0.001$ & i vs. iv \\
\hline $\begin{array}{l}\text { Temporomandibular joint } \\
\text { disorder }\end{array}$ & & 9 & 2.9 & 9 & 7.4 & 6 & 11.5 & 11 & 28.2 & 5 & 21.7 & $<0.001$ & i vs. iii, iv, v \\
\hline he or tension headaches & & 112 & 35.6 & 67 & 54.9 & 26 & 50.0 & 19 & 48.7 & 10 & 43.5 & 0.003 & i vs. ii \\
\hline Irritz & & 7 & & 6 & 4.9 & 5 & 9 & 7 & 17.9 & 5 & 21 & $<0$. & i vs. iv, v \\
\hline Mult & & 3 & & 1 & 0.8 & 1 & & 0 & 0.0 & 0 & 0 & 0.881 & - \\
\hline Neck & & 21 & 6. & 13 & 10.7 & 12 & & 9 & & 9 & & & i vs. iii, iv, v \\
\hline Anx & & 7 & 2 & 8 & & 9 & & & & 5 & & & $\mathrm{v}, \mathrm{v}$ \\
\hline Depression & & 13 & 4.1 & 19 & 15.6 & 10 & & 11 & & 8 & & $<0.001$ & i vs. iii, iv, v \\
\hline $\begin{array}{l}\text { BPI: pain interference score, } n \text {, } \\
\text { mean (SD) }\end{array}$ & 501 & 281 & $1.8(2.2)$ & 113 & $3.0(2.4)$ & 47 & $4.1(2.4)$ & 37 & $4.9(2.9)$ & 23 & $7.2(2.4)$ & $<0.001^{\mathrm{b}}$ & $\begin{array}{l}\text { i vs. ii, iii, iv, v } \\
\text { ii, iii, vs. v }\end{array}$ \\
\hline $\begin{array}{l}\text { BPI: pain severity score, } n \text {, mean } \\
\text { (SD) }\end{array}$ & 516 & 291 & $2.2(2.1)$ & 118 & $3.2(2.2)$ & 47 & $3.9(2)$ & 37 & $4.0(2.1)$ & 23 & $5.1(2.0)$ & $<0.001^{\mathrm{b}}$ & $\begin{array}{l}\text { i vs. ii, iii, iv, v } \\
\text { Ii vs. v } \\
. . .\end{array}$ \\
\hline PHQ-9 score, $n$, mean (SD) & 550 & 314 & $10.9(3.5)$ & 122 & $14.8(5.4)$ & 52 & $17.5(5.7)$ & 39 & $20.8(6.3)$ & 23 & $\begin{array}{l}24.7 \\
(8.3)\end{array}$ & $<0.001$ & $\begin{array}{c}\text { i vs. ii, iii, iv, v } \\
\text { ii vs. iii, iv, v } \\
\text { iii vs. v }\end{array}$ \\
\hline
\end{tabular}

BMI: body mass index; CSI: Central Sensitization Inventory; BPI: Brief Pain Inventory; PHQ: Patient Health Questionnaire. ${ }^{\mathrm{a} U}$ Using a chi-square test or oneway analysis of variance (ANOVA) and post hoc test with residual analysis and Benjamini-Hochberg test for a chi-square test and Bonferroni test for ANOVA were performed. ${ }^{b}$ Using a Kruskal-Wallis test, a post hoc test with the Bonferroni test was performed. ${ }^{\mathrm{c}}$ Missing values were excluded

mean age was significantly higher in the subclinical score group than in the mild and severe score groups. The number of 10 CSS-related diseases, BPI pain interference and severity scores, and PHQ-9 score of the mild, moderate, severe, and extreme score groups were significantly higher than those of the subclinical score group (Table 3).

ANCOVA followed by Bonferroni's test, after adjustments for confounding factors with sex, age, BMI, smoking, alcohol, and caffeine intake and the number of CSI-B or BPI or PHQ-9, showed that the CSI-A subclinical group had lower BPI pain interference and severity scores. Figures 1(a) and 1(b)), PHQ-9 (Figure 1(c)), and a smaller number of CSS-related diseases on CSI-B (Figure $1(\mathrm{~d})$ ) than the CSI-A mild, moderate, severe, and extreme groups. The PHQ-9 score, BPI pain interference and severity score, and a number of CSS-related diseases on CSI-B increased significantly with increasing CSI-A scores.

In Table 4, a greater number of CSI-B resulted in higher CSI-A scores $(p<0.001)$. Additionally, compared with the 0 CSS-related disease group on CSI-B, the $1,2,3$, and 4-6 CSS-related disease groups showed significantly higher 


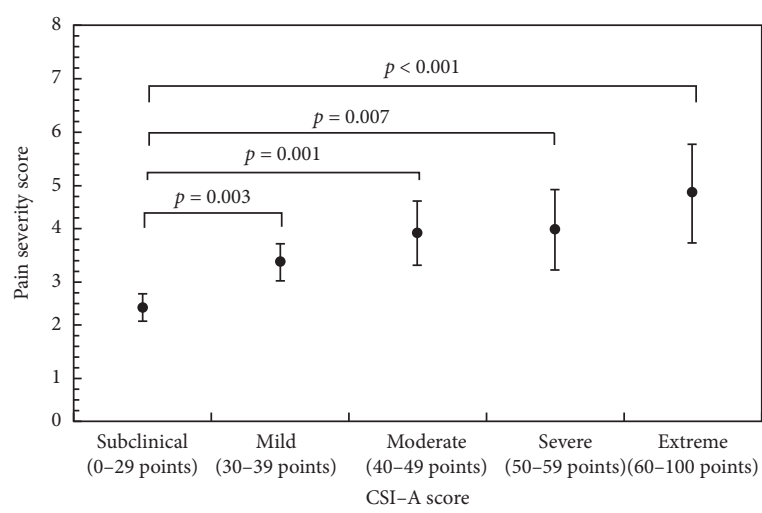

(a)

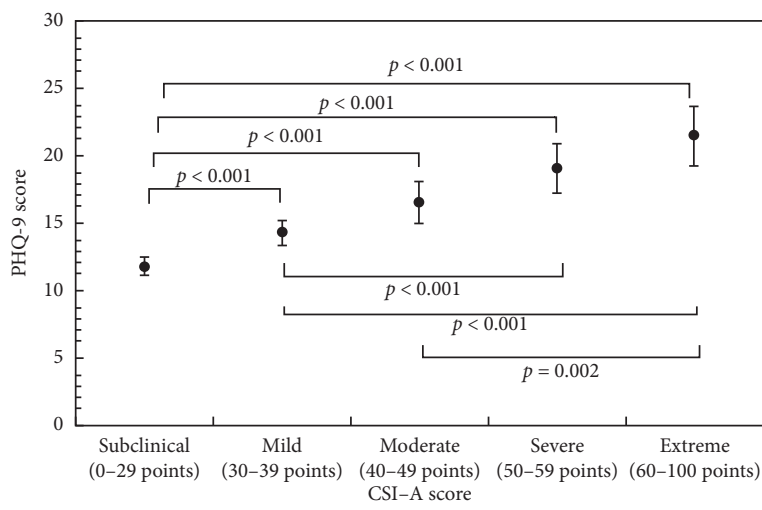

(c)

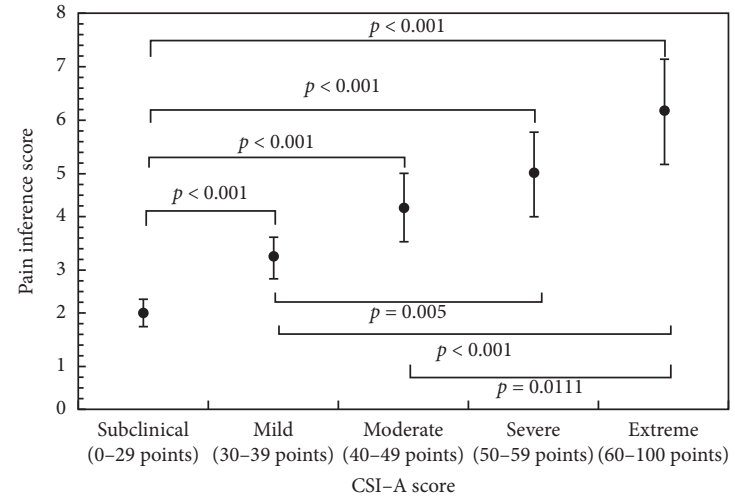

(b)

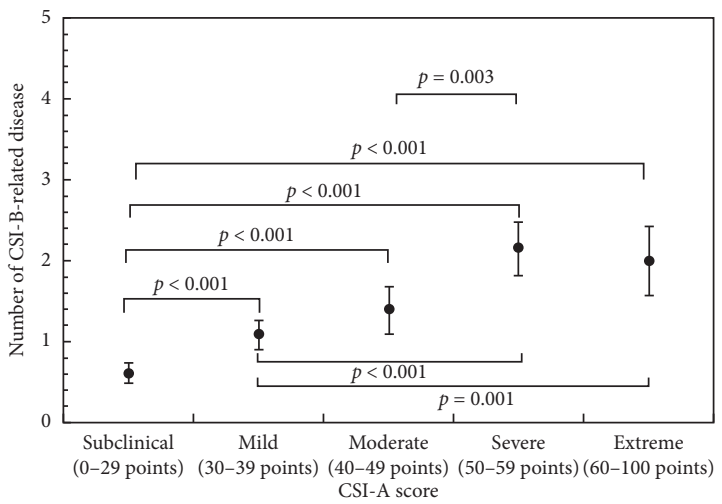

(d)

FIGURE 1: Relationship between clinical parameters and CSI-A score groups in the patients. Error bar indicates the $95 \%$ confidence interval. CSI: Central Sensitization Inventory; PHQ-9: Patient Health Questionnaire. using ANCOVA followed by post hoc comparison with the Bonferroni test. (a) and (b) were adjusted for sex, age, BMI, smoking, alcohol, caffeine and CSS-related diseases on CSI-B. (c) was adjusted for sex, age, BMI, smoking, alcohol, caffeine, CSS-related diseases on CSI-B, and BPI pain interference and severity score. (d) was adjusted for sex, age, BMI, smoking, alcohol, caffeine, PHQ-9 score, and BPI pain severity and interference score. (a) $(n=459),(\mathrm{b})(n=474),(\mathrm{c})$ $(n=452)$, and $(\mathrm{d})(n=452)$.

TABLE 4: Relationship between the number of CSS-related diseases on CSI-B and CSI-A scores.

\begin{tabular}{|c|c|c|c|c|c|c|c|c|c|c|}
\hline \multirow{2}{*}{ CSS-related diseases on CSI-B } & \multirow{2}{*}{$n$} & \multirow{2}{*}{$\%$} & \multicolumn{4}{|c|}{ CSI-A score } & \multicolumn{4}{|c|}{ CSI-A score (more than 40 points) } \\
\hline & & & Mean $^{\mathrm{b}}$ & SE & $p$ value $^{\mathrm{a}}$ & Post hoc test ${ }^{\mathrm{c}}$ & $\mathrm{OR}^{\mathrm{d}}$ & $95 \%$ CI & $p$ value & $p$ for trend \\
\hline 0 diseases & 178 & 39.4 & 23.9 & 0.8 & & 0 vs. $1,2,3,4-6$ & Ref. & & & \\
\hline 1 disease & 171 & 37.8 & 29.5 & 0.8 & & 1 vs. $2,3,4-6$ & 2.70 & $1.15,6.34$ & 0.023 & \\
\hline 2 diseases & 63 & 13.9 & 34.7 & 1.3 & $<0.001$ & 2 vs. $4-6$ & 4.86 & $1.78,13.25$ & 0.002 & $<0.001$ \\
\hline 3 diseases & 25 & 5.5 & 36.3 & 2.1 & & & 20.09 & $5.16,78.20$ & $<0.001$ & \\
\hline 4-6 diseases & 15 & 3.3 & 43.2 & 2.7 & & & 38.59 & $6.97,213.58$ & $<0.001$ & \\
\hline
\end{tabular}

CSS = central sensitization syndrome; CSI = Central Sensitization Inventory. ${ }^{a}$ Using a general linear model with ANCOVA; ${ }^{b}$ after adjustment for sex, age, BMI, smoking, alcohol, caffeine, BPI pain severity score, BPI pain interference score, and PHQ-9 score; ${ }^{c}$ using a Bonferroni test $(p<0.05)$; ${ }^{\mathrm{d}}$ using a multivariable logistic model after adjusting for sex, age, BMI, smoking, alcohol, caffeine, BPI pain severity score, BPI pain interference score, and PHQ-9 score.

odds ratios (ORs). The $p$ value for the trend test was $<0.001$ among 5 CSS-related disease groups classified according to the cutoff point $(\geq 40)$ of CSI-A scores.

\section{Discussion}

In this study, we assessed symptoms related to CSS in patients with neurological, psychological, and pain disorders in a multicenter case-controlled setting. First, we showed that the CSI-A score and the number of CSS- related diseases on CSI-B were significantly greater in the patient group than in the control group. In addition, we calculated that the effect sizes for CSI-A and CSI-B between the patient and control groups were large. Next, we found that pain severity, the degree of pain interference with daily activities, and depressive symptoms worsened, and a number of CSS-related diagnoses increased as the CSI-A score increased across various pain-related conditions. Previously, the CSI-A score was related to pain intensity and pain interference in patients with 
musculoskeletal disorders [19]. Similar to our study results, the relationship of higher CSI-A severity with an increased comorbid CSS-related diagnosis and CSS-related symptoms, such as pain intensity, anxiety, depressive symptoms, disability, and sleep disturbances, were described in 763 patients with chronic spinal pain disorder [24]. In a study including 20 patients with musculoskeletal pain, meditation analysis found that the relationship among anxiety symptoms, depression symptoms, and pain intensity was totally mediated by central sensitization [25]. However, to the best of our knowledge, there have been no large sample studies of neuropsychiatric and pain disorders, including Parkinson's disease, migraine, and restless legs syndrome; psychological diseases, including depression; and various pain disorders with an appropriate control group, investigating CSS-related symptoms.

A large population-based study consisting of 8,930 respondents on a health survey showed that women reported poorer health scores than men did, particularly those aged between 30 and 40 years and over 70 years [26]. Therefore, to address the possible effect of age and sex on the CSI-A score and its impact, we used ANCOVA to adjust for age and sex to analyze all the relationships between CSIA severity and clinical factors. In a study analyzing 1,160 sequelae of 289 diseases and injuries, among leading causes of years lived with disability, the main contributors to global years lived with disability were mental and behavioral disorders and musculoskeletal disorders. Low back pain, major depressive disorder, neck pain, other musculoskeletal disorders, anxiety disorders, and migraine, which were included in our study, were ranked within the top 10 causes [27]. Thus, in this questionnaire-based, multicenter survey, it was important to assess the impact of central sensitization in 551 Japanese patients with various painrelated diseases.

Our finding that, as the CSI-A score increased, painrelated disability and depressive symptoms worsened even after adjustment for CSS-related diseases on CSI-B suggests that this impact of central sensitization was not specific to certain diseases. Alternatively, central sensitization may relatively uniformly impact pain-related disability and psychiatric symptoms in various pain-related conditions. Klyne et al. [28] reported signs of central sensitization in acute low back pain recovery in many patients, but it may result in poor outcomes when combined with other psychological factors. Additionally, we suggest that central sensitization may play a role in the shared pathophysiology of these conditions. Among neurological diseases, in migraine, central sensitization may play a role in trigeminal nerve activation and cortical spreading depression [11]. The neuropeptide calcitonin gene-related peptide is abundantly found in the trigeminal ganglion, and its release from the peripheral terminals may participate in the enhancement of central sensitization [29]. Hyperexcitability of the central nervous system is a core mechanism of central sensitization. Reduced pain inhibition and activation of NDMA receptors in the central nervous system also contribute to persistent chronic pain, producing abnormal sensations such as allodynia and hyperalgesia [30].

We found a significant link between higher depressive symptoms and higher CSS burden (CSI-A scores) in this study. Brain limbic structures are innervated by serotoninergic median raphe neurons and noradrenergic neurons from the locus coeruleus, in which dysregulation of these neurons likely mediates features of psychiatric diseases such as depression and anxiety [31]. Thus, it is possible that altered, long-term central stimulation to brain regions, including the limbic system, can induce and enhance persistent central sensitization, contributing to chronic painful conditions in various pain-related diseases. Therefore, the management of central sensitization could be an important target for refractory pain-related diseases.

The limitations of this study include a cross-sectional design in patient analysis, and CSS-related diagnoses were made by participants with CSI-B and not by physicians. Relatively few participants had more than 3 CSS-related diseases; thus, the $95 \% \mathrm{CI}$ of the OR among the 3 and $4-6$ CSS-related disease groups was wide. However, because the trend test for the OR among the 5 CSS-related disease groups showed significant differences, a relationship between the number of CSS-related diseases and a CSI-A score of $\geq 40$ was more likely. Regarding potential confounding factors for lifestyle, smoking, alcohol, and caffeine intake were the only options for multivariate analysis. Further prospective studies and more confounding factors are warranted to show a longitudinal impact of CSS on disease progression in a patient group. Finally, we used a validated questionnaire to assess CSS-related symptoms (CSI) [32]; however, CSI does not identify and measure central sensitization itself, and currently, there have been no validated tools to identify central sensitization. Additionally, its associations with central sensitization as assessed by experimental measures such as hyperalgesia, facilitated temporal summation, and impaired conditioned pain modulation have not yet been made [8].

\section{Conclusion}

We revealed that CSS-related symptoms were associated with pain severity, pain interference with daily activities, and depressive symptoms in various pain-related diseases in a multicenter setting after adjusting for confounders. Our findings suggest that CSS may participate in these conditions as common pathophysiology and may be a target for future treatment of these disorders.

\section{Data Availability}

The datasets from this study are available from the corresponding author upon reasonable request.

\section{Conflicts of Interest}

The authors declare that there are no conflicts of interest. 


\section{Authors' Contributions}

KS, YH, GK, SY, and KH conceived and designed the study. $\mathrm{KS}, \mathrm{YH}, \mathrm{GK}, \mathrm{TS}, \mathrm{KU}, \mathrm{SY}$, and $\mathrm{KH}$ recruited the participants. KS drafted the paper. $\mathrm{YH}$ and $\mathrm{GK}$ performed the data analysis. YH, GK, SY, TS, KU, and $\mathrm{KH}$ contributed to the interpretation and critically reviewed the manuscript. All the authors read and approved the final version of the manuscript.

\section{Acknowledgments}

The authors would like to thank Ms. Sanae Tani and Ms. Kaori Soma, Department of Neurology, Dokkyo Medical University, for their help with this study. This work was supported by Grants in the Explanation of a Role of the Central Sensitization in the Refractory Disease Patients with Various Type of Symptoms and an Improvement of the Patients Care to Follow it, Research on Policy Planning and Evaluation for Rare and Intractable Diseases; and Health, Labour and Welfare Sciences Research Grants, the Ministry of Health, Labour and Welfare, Japan. The collaborators of this study are Mio Shinozaki, M.D. (Department of Anesthesiology, Dokkyo Medical University School of Medicine); Yoshiyuki Kimura, M.D. (Department of Anesthesiology, Dokkyo Medical University School of Medicine); Shinsuke Hamaguchi, M.D. (Department of Anesthesiology, Dokkyo Medical University School of Medicine); Yuki Numata, M.D. (Department of Anesthesiology, Dokkyo Medical University School of Medicine); Makoto Komatsuzaki, M.D. (Department of Anesthesiology, Dokkyo Medical University School of Medicine); Yuya Sato, M.D. (Department of Anesthesiology, Dokkyo Medical University School of Medicine); Yoshinori Okubo, M.D. (Okubo Clinic); Yukio Midorikawa, M.D. (Dokkyo Medical University, Nikko Medical Center); Hirotoshi Egawa, M.D. (Egawa Pain Clinic); Toshiya Kobayashi, M.D. (Sano Kosei General Hospital); Yoshinori Saeki, M.D. (Department of Psychiatry, Dokkyo Medical University); Sachiko Kase, M.D. (Japanese Red Cross Koga Hospital); Tetsuo Takiguchi, M.D. (Pain Ichijo Clinic); Masayuki Shinohara, M.D. (Mibu East Clinic); Tatsurai Cho, M.D. (Moka Seibu Clinic); Tsutomu Matsumoto, M.D. (Koyanagi Pain Clinic); and Toshimitsu Kitajima, M.D. (Washiya Hospital).

\section{References}

[1] A. Latremoliere and C. J. Woolf, "Central sensitization: a generator of pain hypersensitivity by central neural plasticity," The Journal of Pain, vol. 10, no. 9, pp. 895-926, 2009.

[2] K. L. Petersen, H. L. Fields, J. Brennum, P. Sandroni, and M. C. Rowbotham, "Capsaicin evoked pain and allodynia in post-herpetic neuralgia," Pain, vol. 88, no. 2, pp. 125-133, 2000.

[3] J. Chen and H.-S. Chen, "Pivotal role of capsaicin-sensitive primary afferents in development of both heat and mechanical hyperalgesia induced by intraplantar bee venom injection," Pain, vol. 91, no. 3, pp. 367-376, 2001.

[4] H. Gottrup, G. Juhl, A. D. Kristensen et al., "Chronic oral gabapentin reduces elements of central sensitization in human experimental hyperalgesia," Anesthesiology, vol. 101, no. 6, pp. 1400-1408, 2004.

[5] M. Meeus and J. Nijs, "Central sensitization: a biopsychosocial explanation for chronic widespread pain in patients with fibromyalgia and chronic fatigue syndrome," Clinical Rheumatology, vol. 26, no. 4, pp. 465-473, 2007.

[6] C. J. Woolf, "Evidence for a central component of post-injury pain hypersensitivity," Nature, vol. 306, no. 5944, pp. 686-688, 1983.

[7] S. E. Harte, R. E. Harris, and D. J. Clauw, "The neurobiology of central sensitization," Journal of Applied Biobehavioral Research, vol. 23, no. 2, Article ID e12137, 2018.

[8] L. Arendt-Nielsen, B. Morlion, S. Perrot et al., "Assessment and manifestation of central sensitisation across different chronic pain conditions," European Journal of Pain, vol. 22, no. 2, pp. 216-241, 2018.

[9] K. A. Sluka and D. J. Clauw, "Neurobiology of fibromyalgia and chronic widespread pain," Neuroscience, vol. 338, pp. 114-129, 2016.

[10] A. E. Williams, M. M. Miller, E. J. Bartley, K. M. McCabe, K. L. Kerr, and J. L. Rhudy, "Impairment of inhibition of trigeminal nociception via conditioned pain modulation in persons with migraine headaches," Pain Medicine, vol. 20, no. 8, pp. 1600-1610, 2019.

[11] M. de Tommaso and V. Sciruicchio, "Migraine and central sensitization: clinical features, main comorbidities and therapeutic perspectives," Current Rheumatology Reviews, vol. 12, no. 2, pp. 113-126, 2016.

[12] K. Stiasny-Kolster, W. Magerl, W. H. Oertel, J. C. Moller, and R. D. Treede, "Static mechanical hyperalgesia without dynamic tactile allodynia in patients with restless legs syndrome," Brain, vol. 127, no. Pt 4, pp. 773-782, 2004.

[13] G. N. Verne and D. D. Price, "Irritable bowel syndrome as a common precipitant of central sensitization," Current Rheumatology Reports, vol. 4, no. 4, pp. 322-328, 2002.

[14] F.-Y. Chang and C.-L. Lu, "Irritable bowel syndrome and migraine: bystanders or partners?" Journal of Neurogastroenterology and Motility, vol. 19, no. 3, pp. 301-311, 2013.

[15] S. Grassini and S. Nordin, "Comorbidity in migraine with functional somatic syndromes, psychiatric disorders and inflammatory diseases: a matter of central sensitization?" Behavioral Medicine, vol. 43, no. 2, pp. 91-99, 2017.

[16] D. Zarcone and S. Corbetta, "Shared mechanisms of epilepsy, migraine and affective disorders," Neurological Sciences, vol. 38, no. S1, pp. 73-76, 2017.

[17] J. Van Oosterwijck, J. Nijs, M. Meeus, and L. Paul, "Evidence for central sensitization in chronic whiplash: a systematic literature review," European Journal of Pain, vol. 17, no. 3, pp. 299-312, 2013.

[18] T. G. Mayer, R. Neblett, H. Cohen et al., "The development and psychometric validation of the central sensitization inventory," Pain Practice, vol. 12, no. 4, pp. 276-285, 2012.

[19] K. Tanaka, T. Nishigami, and A. Mibu, "Validation of the Japanese version of the Central Sensitization Inventory in patients with musculoskeletal disorders," PLoS One, vol. 12, no. 12, Article ID e0188719, 2017.

[20] R. Neblett, M. M. Hartzell, T. G. Mayer, H. Cohen, and R. J. Gatchel, "Establishing clinically relevant severity levels for the central sensitization inventory," Pain Practice, vol. 17, no. 2, pp. 166-175, 2017.

[21] J. Uki, T. Mendoza, C. S. Cleeland, Y. Nakamura, and F. Takeda, "A Brief cancer pain assessment tool in Japanese," Journal of Pain and Symptom Management, vol. 16, no. 6, pp. 364-373, 1998. 
[22] K. Muramatsu, K. Kamijima, M. Otsubo, H. Miyaoka, Y. Muramatsu, and F. Gejyo, "The patient health questionnaire, Japanese version: validity according to the mini-international neuropsychiatric interview-plus," Psychological Reports, vol. 101, no. 3, pp. 952-960, 2007.

[23] J. Cohen, “A power primer," Psychological Bulletin, vol. 112, no. 1, pp. 155-159, 1992.

[24] R. Neblett, M. M. Hartzell, M. Williams, K. R. Bevers, T. G. Mayer, and R. J. Gatchel, "Use of the Central Sensitization Inventory (CSI) as a treatment outcome measure for patients with chronic spinal pain disorder in a functional restoration program," The Spine Journal, vol. 17, no. 12, pp. 1819-1829, 2017.

[25] H. Shigetoh, Y. Tanaka, M. Koga, M. Osumi, and S. Morioka, "The mediating effect of central sensitization on the relation between pain intensity and psychological factors: a crosssectional study with mediation analysis," Pain Research and Management, vol. 2019, Article ID 3916135, 6 pages, 2019.

[26] M. Sullivan and J. Karlsson, "The Swedish SF-36 health survey III. Evaluation of criterion-based validity," Journal of Clinical Epidemiology, vol. 51, no. 11, pp. 1105-1113, 1998.

[27] T. Vos, A. D. Flaxman, M. Naghavi et al., "Years lived with disability (YLDs) for 1160 sequelae of 289 diseases and injuries 1990-2010: a systematic analysis for the global burden of disease study 2010," Lancet (London, England), vol. 380, no. 9859 , pp. 2163-2196, 2012.

[28] D. M. Klyne, G. L. Moseley, M. Sterling, M. F. Barbe, and P. W. Hodges, "Are signs of central sensitization in acute low back pain a precursor to poor outcome?" Journal of Pain, vol. 20, no. 8, pp. 994-1009, 2019.

[29] S. Iyengar, K. W. Johnson, M. H. Ossipov, and S. K. Aurora, "CGRP and the trigeminal system in migraine," Headache: The Journal of Head and Face Pain, vol. 59, no. 5, pp. 659-681, 2019.

[30] C. den Boer, L. Dries, B. Terluin et al., "Central sensitization in chronic pain and medically unexplained symptom research: a systematic review of definitions, operationalizations and measurement instruments," Journal of Psychosomatic Research, vol. 117, pp. 32-40, 2019.

[31] K. J. Ressler and C. B. Nemeroff, "Role of serotonergic and noradrenergic systems in the pathophysiology of depression and anxiety disorders," Depression and Anxiety, vol. 12, no. S1, pp. 2-19, 2000.

[32] T. Scerbo, J. Colasurdo, S. Dunn, J. Unger, J. Nijs, and C. Cook, "Measurement properties of the central sensitization inventory: a systematic review," Pain Practice, vol. 18, no. 4, pp. 544-554, 2018. 